\title{
MEGARA FOCAL PLANE SUBSYSTEMS
}

\author{
Pérez-Calpena, A. ${ }^{1}$, Arrillaga X. ${ }^{2}$, Gil de Paz, A. ${ }^{3}$, Sánchez-Blanco. E. ${ }^{1}$, García-Vargas, M.L. ${ }^{1}$, \\ Carrera, M.A. ${ }^{2}$, Gallego, J. ${ }^{3}$, Carrasco E. ${ }^{4}$, Sánchez, F.M. ${ }^{5}, V^{\prime}$ lchez, J.M. ${ }^{6}$ \& MEGARA Team \\ ${ }^{1}$ FRACTAL SLNE (Madrid, Spain), ${ }^{2}$ AVS (Guipuzkoa, Spain), ${ }^{3}$ UCM (Madrid, Spain), ${ }^{4}$ INAOE \\ (Puebla, México), ${ }^{5}$ UPM (Madrid, Spain), ${ }^{6}$ IAA (Granada, Spain)
}

\begin{abstract}
MEGARA (Multi-Espectrógrafo en GTC de Alta Resolución para Astronomía) is the future optical Integral-Field Unit (IFU) and Multi-Object Spectrograph (MOS) for GTC. The Fiber Units are placed at one Folded Cassegrain focus and feed the spectrograph located on a Nasmyth-type platform.

This paper summarizes the status of the design of the MEGARA Folded Cassegrain Subsystems after the PDR (held on March 2012), as well as the prototyping that has been carried out during this phase.

The MEGARA Fiber Unit has two IFUs: a Large Compact Bundle covering $12.5 \operatorname{arcsec}$ x 11.3 arcsec on sky (100 microns fiber-core), and a Small Compact Bundle, of $8.5 \operatorname{arcsec}$ x $6.7 \operatorname{arcsec}$ (70 microns fiber-core), plus a Fiber MOS positioner, able to place up to 100 mini-bundles 7 fibers each (100 microns fiber-core) in MOS configuration within a 3.5 arcmin x 3.5arcmin FOV. A field lens provides a telecentric focal plane where the fibers are located. Microlens arrays couple the telescope beam to the collimator focal ratio at the entrance of the fibers (providing the $f / 17$ to $f / 3$ focal ratio reduction to enter into the fibers). Finally, the fibers, organized in bundles, end in the pseudo-slit plate, which will be placed at the entrance focal plane of the MEGARA spectrographs.
\end{abstract}

Keywords: MEGARA, GTC, fiber, microlens, pseudo-slit, robotic positioners.

\section{INTRODUCTION}

MEGARA (Multi-Espectrógrafo en GTC de Alta Resolución para Astronomía) is a fiber-fed spectrograph with two optical Integral-Field Units (IFU) and a set of robotic positioners for Multi-Object Spectroscopy (MOS) that will be installed at Folded-Cassegrain focus of the GTC (Gran Telescopio CANARIAS) 10.4-m telescope. The fibers will feed one (MEGARA-Basic) or two (MEGARA-Advanced) spectrographs to be placed on a Nasmyth-type platform.

The funds from GRANTECAN plus the in-kind contributions from the partners are expected to cover MEGARA-Basic, which includes the construction of the fiber bundles plus one spectrograph. Additional funds from MINECO (Ministerio de Economía y Competitividad, Spain) and/or other sources, could eventually cover the construction of MEGARAAdvanced, implying the provision of a second spectrograph, in this case with the input coming from one of the IFU bundles and the MOS (Dispersed bundle) simultaneously.

The Universidad Complutense de Madrid (UCM) leads the MEGARA Consortium. The other partners that form part of the MEGARA Consortium are the Instituto Nacional de Astrofísica, Óptica y Electrónica (INAOE) in Mexico, the Instituto de Astrofísica de Andalucía (IAA-CSIC) and the Universidad Politécnica de Madrid (UPM). The MEGARA Science Team includes scientists from these institutions and also researchers from other research centers in Spain, Mexico and the University of Florida. This team includes experts from different areas, from exo-planets \& cool stars to cosmology, going through massive stars, planetary nebula, and nearby galaxies.

This paper provides an overview of the MEGARA focal plane subsystems, which are the elements that collect the light at the GTC Folded Cassegrain focus and deliver it to the spectrograph entrance, and the prototypes that have been designed

Ground-based and Airborne Instrumentation for Astronomy IV, edited by lan S. McLean, Suzanne K. Ramsay, Hideki Takami, Proc. of SPIE Vol. 8446, 84465E · @ 2012 SPIE · CCC code: 0277-786X/12/\$18 · doi: 10.1117/12.926068 
and manufactured during the preliminary design phase of the instrument. The MEGARA Preliminary Design Review took place on 21-22 March 2012.

The focal plane elements described in this paper are (a) the Field lens to correct the telecentricity, (b) the microlens arrays that convert the telescope beam into a beam suitable for fiber entrance with minimum loss, (c) the fibers that will collect the light and deliver it to the entrance of the spectrograph(s), (d) the robotic positioners that allows placement of the fiber minibundles at any position in the MOS Field Of View (FOV) and, finally, (e) the fiber arrangement at the pseudo-slit position. All these elements shall be supported by the mechanical supports (Folded Cassegrain Rotator Adapter) needed to attach them to the interface provided by GTC at the Folded Cassegrain Station.

Two prototypes have been manufactured during the preliminary design phase. A prototype of a robotic positioner with fibers, to measure its mechanical behavior, and a second prototype of the fiber link (a fibers minibundle plus microlenses) to measure optical performance. Both prototypes reproduce the real fiber length of 40 meters.

Section 2 is devoted to describe the overall organization of the MEGARA fiber units at the GTC Focal plane. Section 3 describes the MEGARA focal plane elements. Section 4 provides an overview of the MEGARA prototypes manufactured during the preliminary design and summarizes the test results. Finally, Section 5 outlines what is the current status of the MEGARA focal plane elements and the tasks pending to complete the design.

\section{OVERALL DESCRIPTION OF THE FIBER UNITS AT THE FOCAL PLANE}

The MEGARA Instrument is composed of three modes, two IFUs and the MOS mode, which correspond to the three fiber bundles available: the Large Compact Bundle (LCB), the Small Compact Bundle (SCB) and the Dispersed Bundle.

The fiber layout is shown in Fig.1.

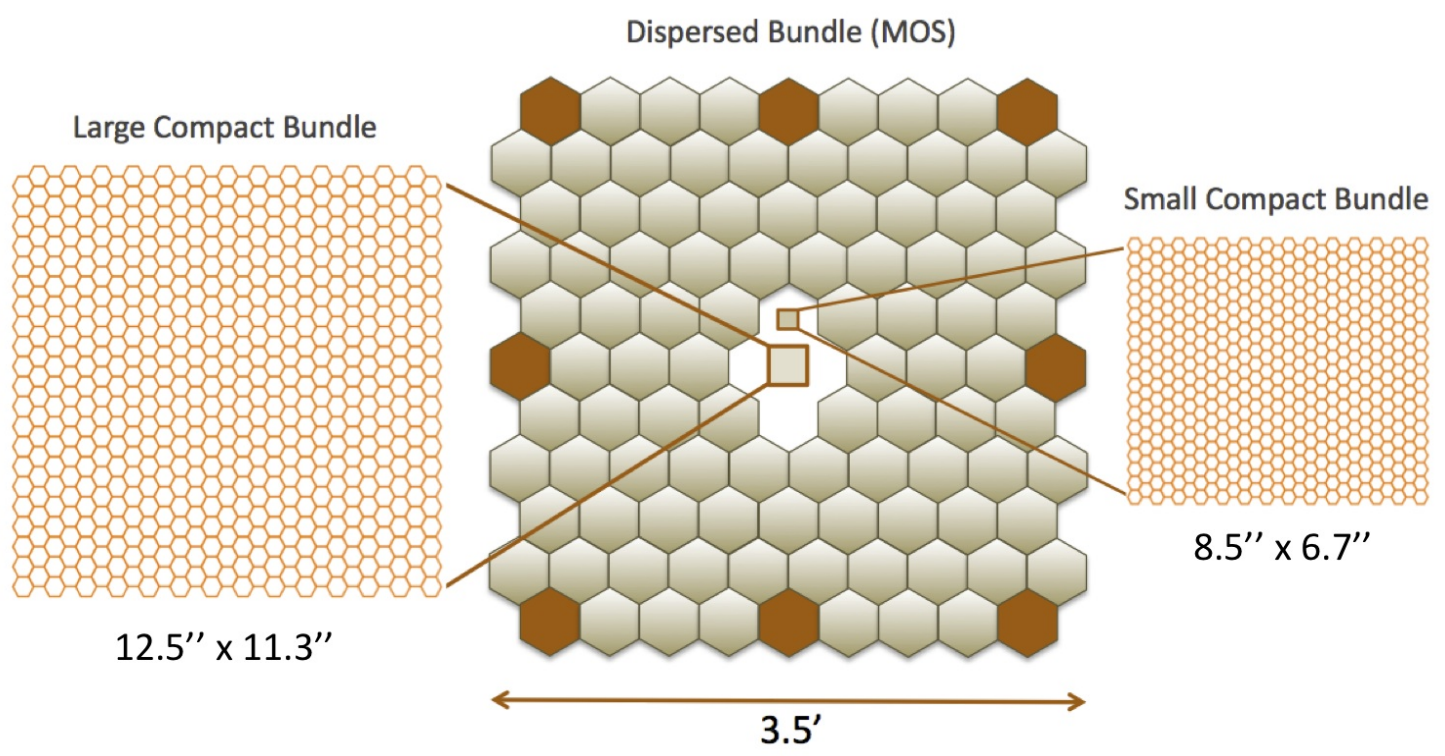

Fig. 1. Layout of the Large and Small Compact (LCB and SCB, respectively) and Dispersed fiber bundles of MEGARA.

\section{- Large Compact Bundle IFU}

The LCB IFU bundle is composed of 567 fibers of $100 \mu \mathrm{m}$ core displayed on an area of 12.5 " x 11.3" near the optical axis of the instrument plus 8 positioner robots (dark orange hexagons in Fig. 1) located in the outer edge of the FOV, used for measuring the sky background simultaneously with the LCB observations.

The $100 \mu \mathrm{m}$ core fibers spaxel size is 0.62 arcsec on sky.

The LCB IFU is ideally suited for the study of individual very compact objects and for absolute flux-calibration purposes when the MOS mode is used. 


\section{- Small Compact Bundle IFU}

The Small Compact Bundle IFU is composed of 500 fibers with a smaller core diameter $(70 \mu \mathrm{m})$. This yields an improved spatial (spaxel size of 0.42 arcsec) and spectral resolution but gives a more limited FOV, 8.5 " x 6.7".

The IFU SCB center is offset approximately 19 arcsec from the center of the LCB.

\section{- MOS Mode (Dispersed bundle)}

The robotic positioners will be able to place individual mini-bundles of fibers ( 7 fibers each) anywhere in the central 3.5 arcmin $\times 3.5$ arcmin around the two IFU bundles. These fibers will go to a different pseudo-slit and, in the case of the MEGARA-Advanced configuration, also to a separate spectrograph. The current configuration includes a total of 100 actuators, 92 of them going to this dedicated MOS-mode pseudo-slit, which would have a number of 644 fibers. The rest of the positioners (8) would be evenly distributed along the pseudo-slit of the LCB IFU. Note that these 8 positioners, since they are devoted to measuring the sky background, are not required to move, so they would be always kept in the parked position. Although we do not exclude the possibility of simply having these eight 7-fiber bundles mechanically attached to the Folded-Cassegrain Rotator Adaptor frame, our current estimate is that, due to the associated increase in design costs, this option could more expensive that having eight additional robotic positioners parked. Besides, having positioners provides more flexibility as they allow us to move the sky-substraction minibundles to a different position in case that a bright star is located at the positioner parking coordinates.

In Fig. 2 we show the 3D view of the positioner system (so called Fiber MOS) with the two IFU bundles in the center and an overview of the Folded Cassegrain Rotator Adapter that provides the support to the focal plane elements.
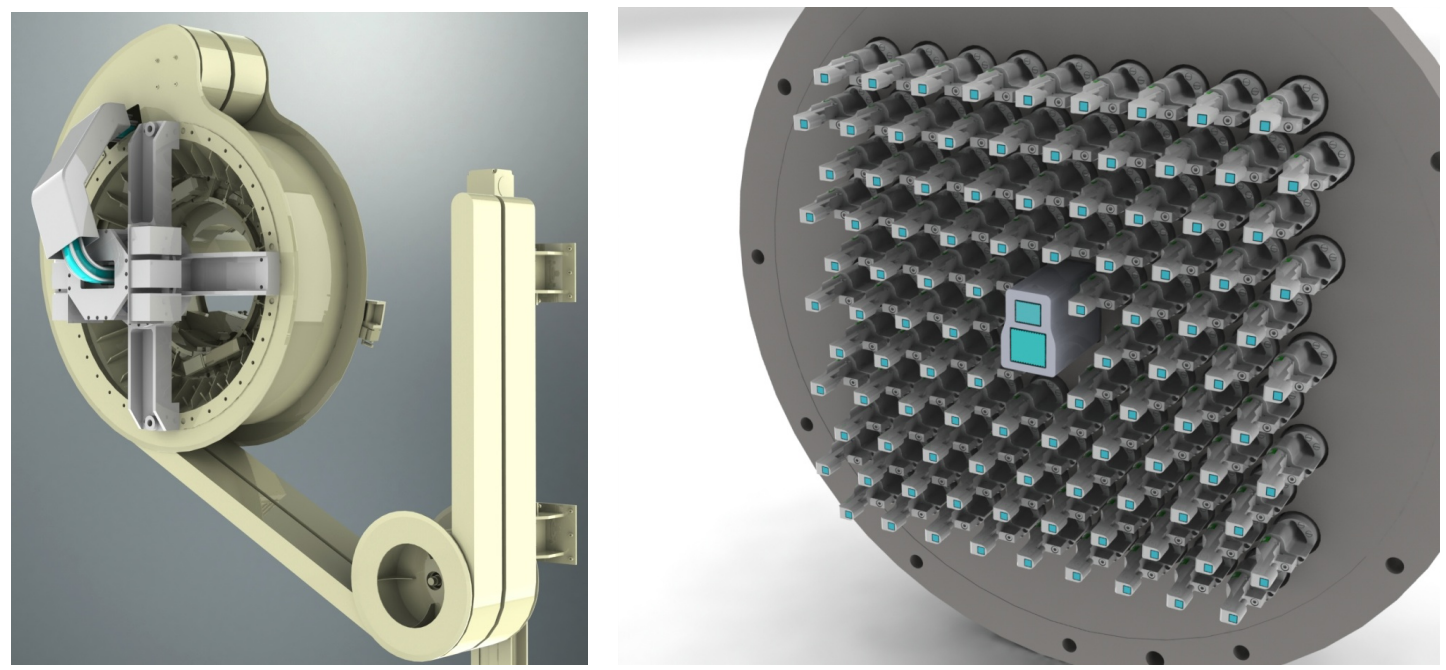

Fig. 2. Folded Cassegrain Rotator Adapter (Left). 3D view of the Fiber MOS system (Right). The positions of the LCB and SCB IFUs are shown as two blue cubes near the center of the system. All positioners are identical.

The fibers constituting the IFUs will be arranged in two pseudo-slits (one for LCB and another one for SCB) while the fibers coming from the robotic positioners (MOS mode) will be arranged in a different pseudo-slit. MEGARA-Basic is composed by a single spectrograph that can be fed either by the LCB or SCB pseudo-slits or the MOS-mode pseudo-slit. MEGARA-Advanced is composed by two spectrographs that can be fed each one by a different bundle, allowing the possibility of simultaneous observations with one of the IFUs and the MOS.

\section{MEGARA FOCAL PLANE SUBSYSTEMS}

This section is devoted to provide an overview of the main MEGARA focal plane elements, which includes (a) the Field lens, (b) the microlens arrays, (c) the fibers, (d) the robotic positioners and (e) the pseudo-slits plates. 


\subsection{Field Lens}

The field lens is added to provide a telecentric field for MEGARA. This is needed because the GTC telescope has the aperture at the secondary mirror and, thus, the exit pupil as seen from the telescope focal plane coincides with this position (-18meters from the focal plane).

The field lens makes the opto-mechanical axes of all the fiber bundles be parallel among them. Thus the positioners move on a flat surface (the focal plane) with their opto-mechanical axis perpendicular to this surface. The field curvature has not disappeared (this would require at least 2 lenses to avoid changing the plate scale) but it is below $0.1 \operatorname{arcsec}$ across the whole FOV.

The field lens will be a fused silica meniscus ( $\mathrm{R} 1=-2147.6, \mathrm{R} 2=-1731.8 \mathrm{~mm}$ and $\mathrm{CT}=30 \mathrm{~mm}$ ) with a diameter of $260 \mathrm{~mm}$ in order to cover the maximum MEGARA FOV 3.5' x 3.5'. Fused silica transmits over the whole wavelength range. Losses are negligible for the lens thickness and are only due to Fresnel.

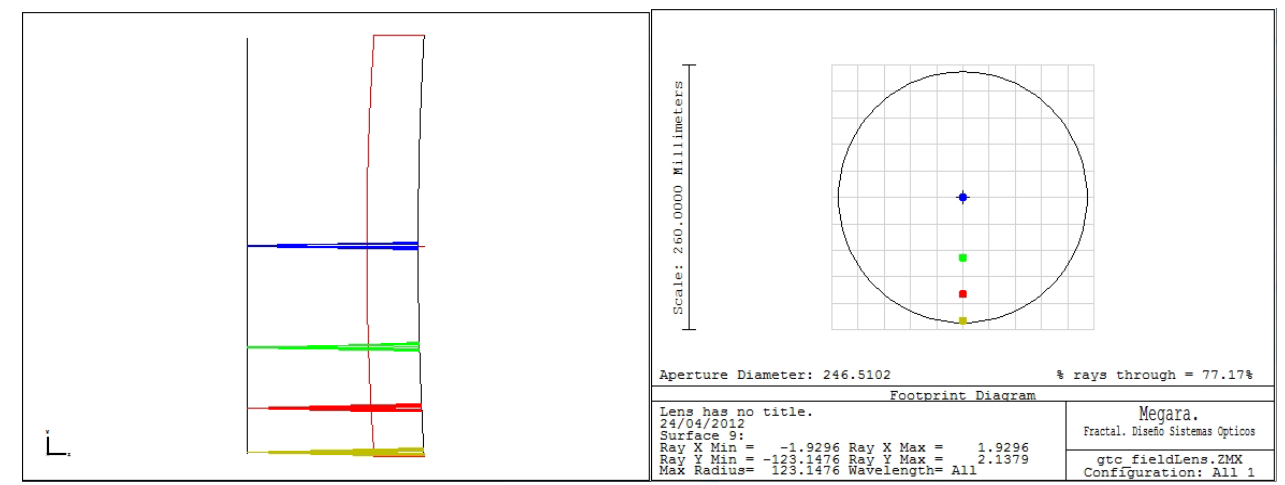

Fig. 3. Field lens layout for the full Megara FOV corrected with the field lens (left). Footprint diagram at the field lens surfaces. The beam cross diameter for the different fields is below $6 \mathrm{~mm}$ diameter (right).

The image quality in a flat surface of the focal plane in the required FOV is given in the Fig. 4. The change with the nominal FOV (without the field lens) is negligible.

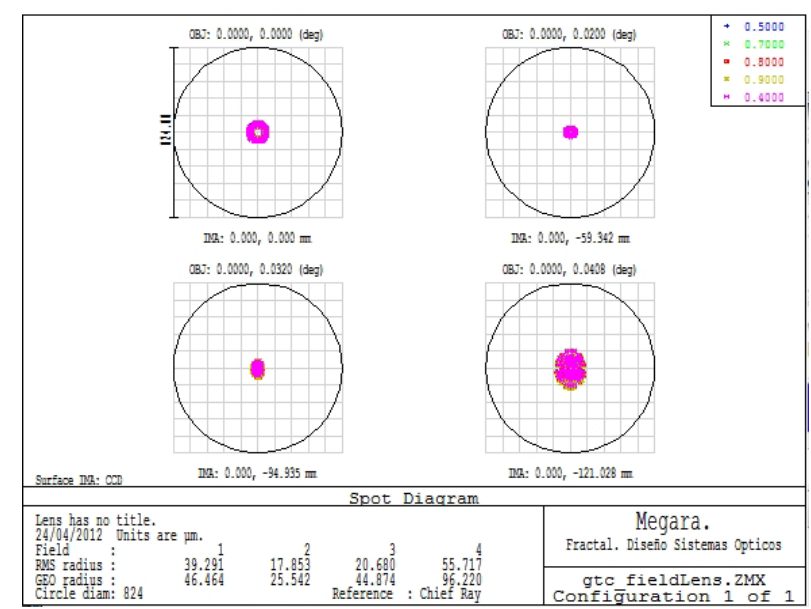

Fig. 4. Spot diagram for the telescope focal plane that is flat. Circle is 1 arcsec.

\subsection{Microlens}

The microlens array defines the FOV that will be seen by a fiber, adapting the telescope $F / \#$ from $f / 17$ to $f / 3$ to minimize focal ratio degradation (FRD) effects during the beam transport and providing a telescope pupil image on the fiber core (needed for stray light control). 
Refractive microlenses are manufactured using a standard semiconductor lithographic technology, which allows very accurate shaping of the lens profile. The microlens array will be placed at the telescope focal plane.

Different microlenses have been designed for LCB/MOS modes $(100 \mu \mathrm{m}$-core fibers) and for SCB mode $(70 \mu \mathrm{m}$-core fibers) and, therefore different arrays for each of the three bundles (LCB, SCB and MOS).

\section{- LCB/MOS microlens design}

In the case of the LCB and MOS the microlenses introduce the telescope light into the $100 \mu \mathrm{m}$-core fibers and, therefore, will have the same optical parameters for both modes although the arrays will be geometrically different (in the number of lenslets used) for the LCB IFU and dispersed-bundle-fed MOS.

The microlens design has been performed looking for a compromise to optimize the flux recovered from point sources with each MEGARA MOS mini-bundle, the fraction of light lost when reimaging the pupil on the fiber core, the need for fully imaging the fiber core in order to preserve the quality of the relative-flux calibration from fiberto-fiber and Differential Atmospheric Refraction effects.

A full suite of tools to simulate all effects both at the level of the sky, considering the pupil re-imaging on the fiber, and on the detector were developed. Fig. 5 shows the results of the simulations carried out to analyze the effects of both on-sky coverage for point sources (when the MOS is used) and of the imaging of the pupil on the fiber core by the microlenses. As can be seen in the upper-right diagram both effects go in opposite directions so an optimum value (regarding these two effects only) is obtained at a spaxel size of 0.56 arcsec approximately.

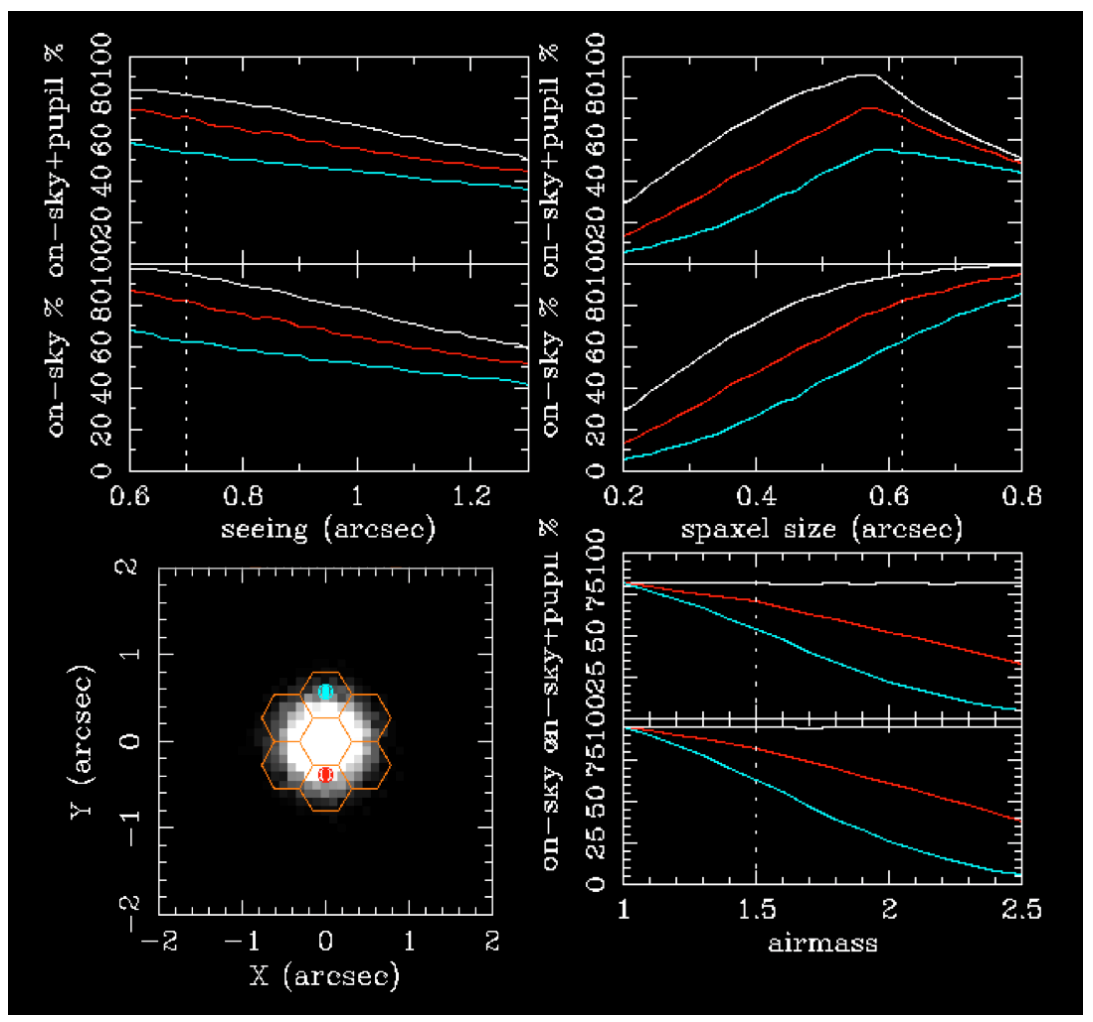

Fig. 5. Results of the $100 \mu \mathrm{m}$-core fibers simulation on the on-sky light distribution as a function of seeing (FWHM; topleft panel), spaxel size (top-right panel) and airmass (bottom-right panel). The 2D distribution of light on the sky in the case of 0.7 " point source using 0.62 spaxels at an airmass of 1.5 is shown at the bottom left. Red (down) and blue (up) dots represent the position of the centroid of the light at the extremes of the LR-U wavelength range at an airmass of 1.5.

However, we should now also consider the effects of both differential Atmospheric Refraction and of stability of the relative-flux calibration. These two latter effects worsen as we reduce the spaxel size. Thus, we decided to increase that spaxel size to 0.62 arcsec per spaxel, still very close to the peak of maximum flux recovered but 
also reducing differential Atmospheric Refraction effects and ensuring a good and stable relative-flux calibration even if the Instrument Calibration Module (ICM) did not provide a perfect f/17 beam.

In addition, there is another issue that plays a role in this trade-off and this is the requirement on the fluxcalibration stability of the system induced by potential fiber-to-fiber misalignments between the pupil images on each fiber core. This should be minimized $(<10 \%$ in the fiber-to-fiber variation of the fraction of the pupil imaged) to ensure a proper relative-flux calibration and cosmetics. This is particularly important should an $\mathrm{f} / 17$ beam not be available from the ICM.

The conclusion from this analysis is that a spaxel size of 0.62 arcsec provides an excellent trade-off among all these effects. This yields a tolerance in the positioning of the image of the pupil on the fiber core of $10 \mu \mathrm{m}$, which should be fulfilled by the planned centering and gluing procedure.

The microlens design in the case of the LCB and MOS is shown in Fig. 6. As also shown in the figure, the spaxel size given here is that of the maximum dimension of the hexagonally-shaped microlens.
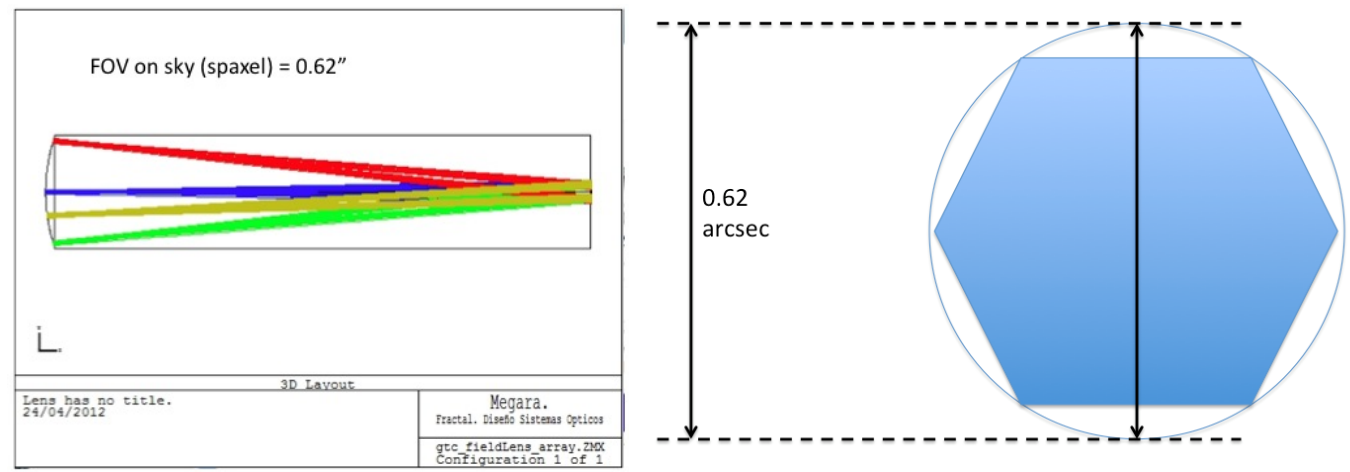

Fig. 6. Detail of the LCB/MOS microlens design showing the telescope focal plane and the FOV aperture (Left). Scheme showing the spaxel size relative to the circle where this spaxel is inscribed (Right).

The oversize of the pupil on the fiber core will decrease $17.8 \%$ the total flux of the maximum telescope aperture, as shown in the figure below.
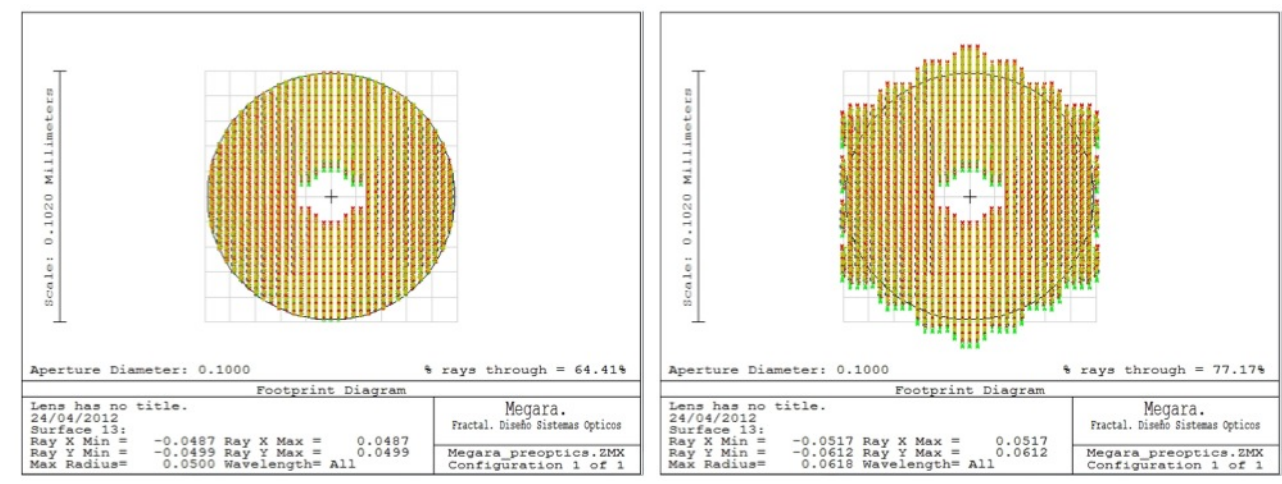

Fig. 7. GTC pupil at the fiber entrance. On the left, the light entering in the fiber. On the right, the complete pupil.

Microlens shall be made in Fused Silica due to its good UV transmission. They shall be aspherical microlenses with the following parameters: ROC $0.844 \mathrm{~mm}$, thickness $2.727 \mathrm{~mm}$, aperture $511 \mu \mathrm{m}$, conic constant -0.9797 .

\section{- SCB Microlens design}

In the case of the SCB the microlenses introduce the telescope light into the $70 \mu \mathrm{m}$ core fibers. 
Fig. 8 below shows the results of the simulations carried out to analyze the effects of both on-sky coverage for point sources and of the imaging of the pupil on the $70 \mu \mathrm{m}$-core fiber by the microlenses. Note, however, that these simulations are done in the context of the evaluation of the feasibility of using such fibers on the Fiber MOS. Regarding their use on the SCB we must take into account that in that case neither the fraction of light recovered from a point source nor different Atmospheric Refraction effects are an issue although, on the other hand, the need of having a somewhat oversized pupil is here more demanding than for the $100 \mu \mathrm{m}$-core fibers. It is because of this that for the SCB IFU the use of 0.42 spaxels (closer to the peak where pupil light losses are negligible) is found as the most optimal design trade-off.

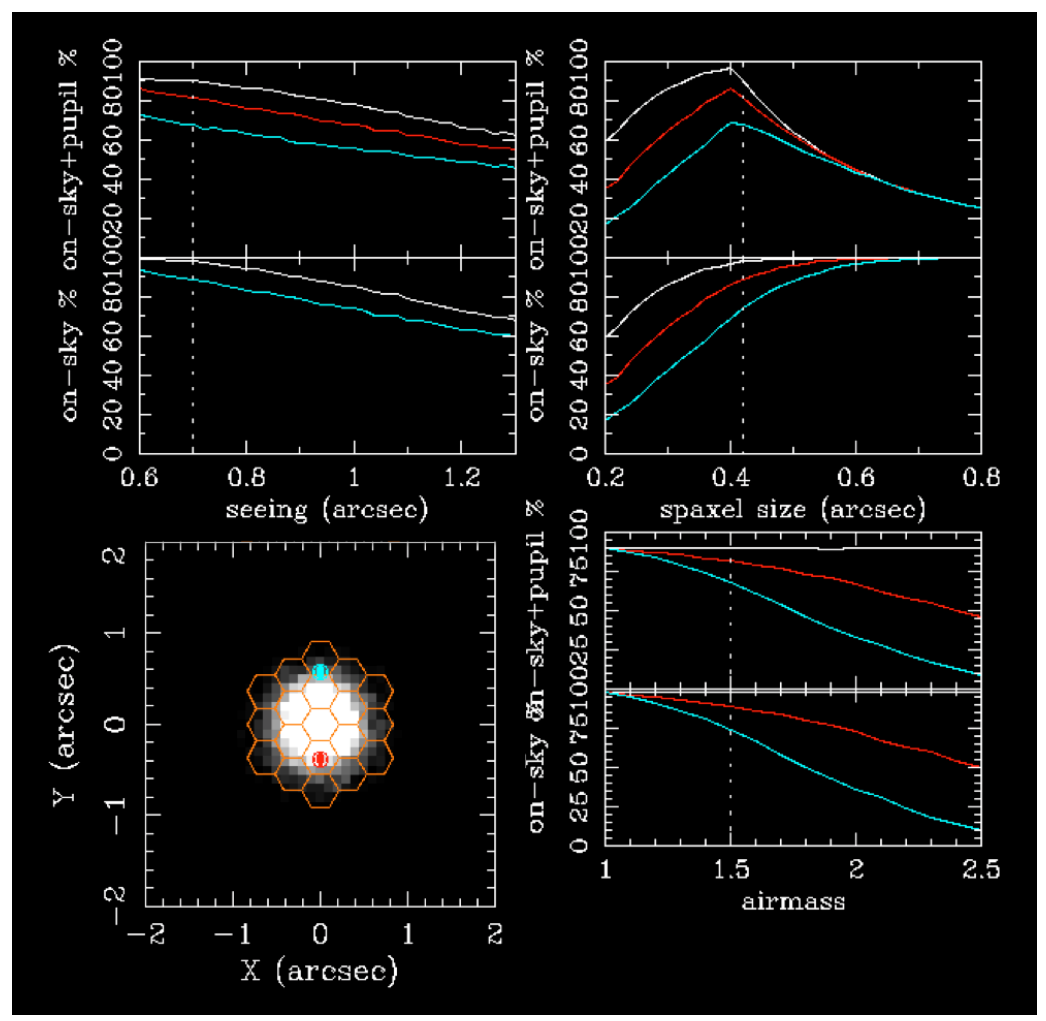

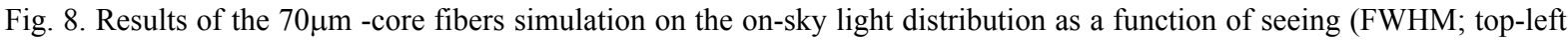
panel), spaxel size (top-right panel) and airmass (bottom-right panel). The 2D distribution of light on the sky in the case of 0.7 -arcsec point source using 0.42 spaxels at an airmass of 1.5 is shown at the bottom left. Red (down) and blue (up) dots represent the position of the centroid of the light at the extremes of the LR-U wavelength range at an airmass of 1.5.

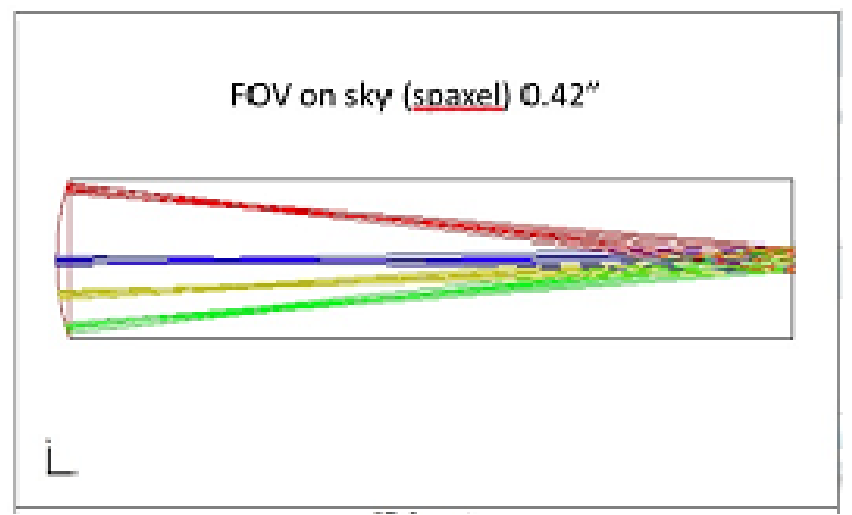

Fig. 9. Detail of the SCB microlens design showing the telescope focal plane and the FOV aperture. 
The microlens design for the SCB is shown in Fig. 9. As in the LCB case, the spaxel size given here is that of the maximum dimension of the hexagonally-shaped microlens.

The oversize of the pupil on the fiber core will decrease $8.8 \%$ the total flux of the maximum telescope aperture, as shown in Fig. 10. This selection yields a tolerance in the positioning of the image of the pupil on the fiber core of $10 \mu \mathrm{m}$, which is the same tolerance used for the $100 \mu \mathrm{m}$-core fibers.

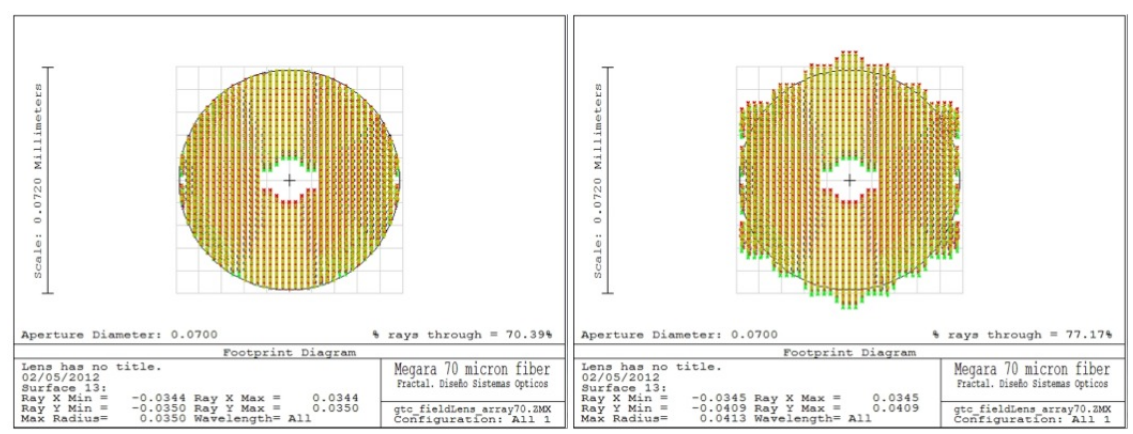

Fig. 10. GTC pupil at the fiber entrance. On the left, the light entering in the fiber. On the right, the complete pupil.

Microlens shall be made in Fused Silica due to its good UV transmission. They shall be aspherical microlens with the following parameters: ROC $0.563 \mathrm{~mm}$, thickness $1.815 \mathrm{~mm}$, aperture $346 \mu \mathrm{m}$, conic constant -0.927 .

\section{- LCB, SCB and MOS Microlens array design}

Microlens arrays must be designed for each mode (LCB, SCB and MOS).

In the arrays the microlenses will be arranged in a hexagonal geometry to maximize the area to be covered.

The manufacturing process requires a real boundary between two lens surfaces. This small region (below $10 \mu \mathrm{m})$ scatters the light to undesired positions. This light is lost. These regions are typically masked to avoid this effect.

Finally the arrays shall contain two fiduciary marks that are referenced to the lens centers within $5 \mu \mathrm{m}$ in order to help for the alignment between the fibers and the array. Fig. 11 shows the design drawing details of the LCB, SCB and MOS microlens array.

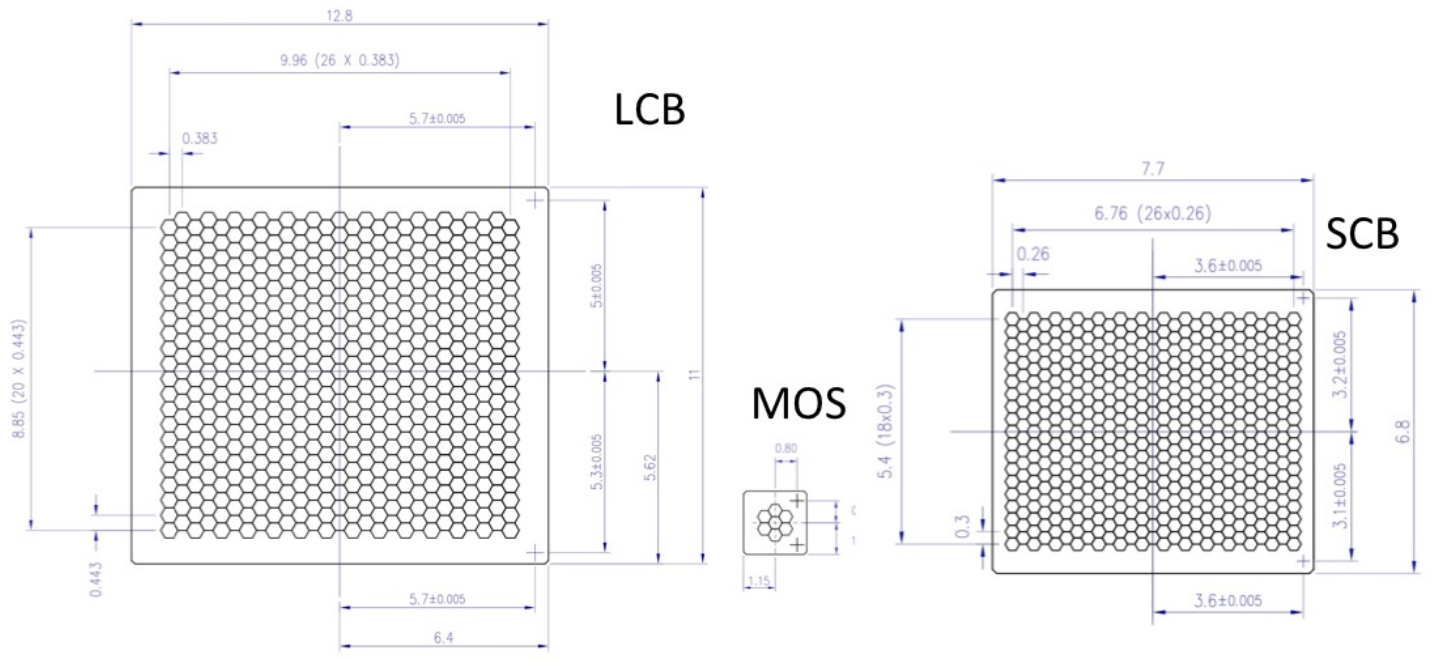

Fig. 11. LCB, MOS and SCB Microlens arrays drawings details. 


\subsection{Fibers}

The fiber cables transport the light from the focal plane to the spectrographs at the pseudo-slit position. The characteristics of the fibers to be used for LCB/MOS and SCB are slightly different.

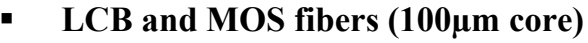

The fiber selected in this case is the one from Polymicro FBP 100/140/170; with a core of $100 \mu \mathrm{m}, 140 \mu \mathrm{m}$ is the cladding and $170 \mu \mathrm{m}$ is the mechanical coating. This fiber has a numerical aperture of $0.20 \pm 0.02$ (optical angle acceptance and output light angle of the fiber, $\sin \left(12.71^{\circ}\right)$ ). This is a wide broadband fiber and provides a good FRD. We shall use this fiber for both the LCB IFU and MOS modes.

Fig. 12 shows the expected transmission for the fiber model Polymicro 100/140/170.

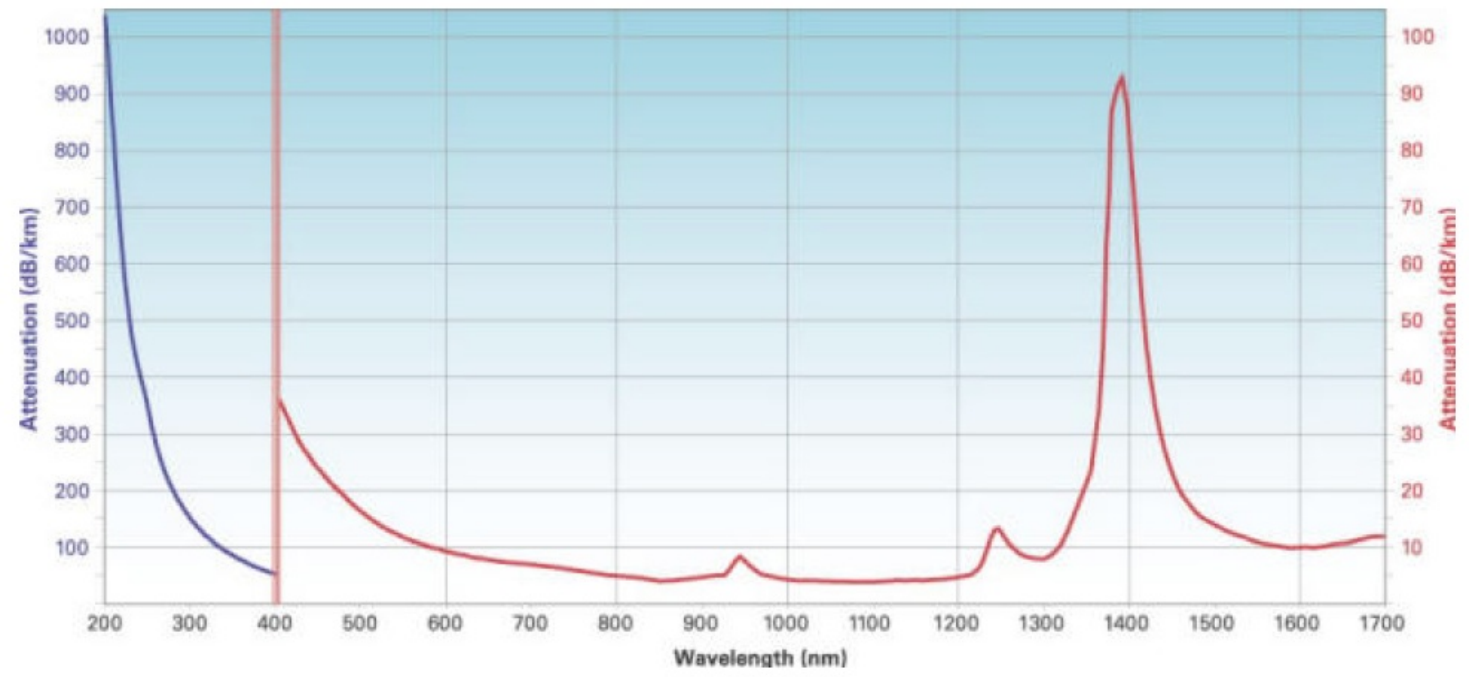

Fig. 12. Fiber model FBP from Polymicro.

Light is collected at the fiber exit at $\mathrm{f} / 3$ to minimize losses due to FRD.

The Dispersed-bundle fibers must be integrated in sub-units of 7 fibers. The LCB fibers shall be all grouped in a single large tube, which shall have octopus geometry and shall be divided into small sub-units where the fibers coming to each pseudo-slit box shall be included. Loose polyurethane tubing (not a tight jacket) will be used to cover all sub-units. Polyurethane material is also the best one to use when a robot is moving. It has very stable dielectric properties over a wide temperature range. It is also abrasion-resistant.

\section{- SCB fibers $(70 \mu \mathrm{m}$ core $)$}

The fibers to be used in the Small Compact Bundle (SCB) share the same manufacturer (Polymicro) and some basic properties with those used for the LCB IFU and MOS modes, such as the numerical aperture and expected transmittance. These fibers will have an external diameter of $170 \mu \mathrm{m}$, identical to the $100 \mu \mathrm{m}$-core fibers fibers.

The Focal Ratio Degradation shall be the same applicable to the $100 \mu \mathrm{m}$ fiber used in the LCB and MOS modes. Thus, we foresee a loss below $5 \%$ of the beam introducing the light at $\mathrm{f} / 17$ and collecting it at $\mathrm{f} / 3$.

Fibers must be also arranged in sub-units as in the LCB and Dispersed bundles. Again, all sub-units will be covered by loose polyurethane tubing and not with a tight jacket. This protection tubing extends along the full bundle length (telescope to spectrograph) to protect the fibers. Polyethylene (PE) is the standard jacket material for outdoor fiber optic cables. PE has excellent moisture and weather-resistance properties. 


\subsection{Fiber MOS positioners}

The Fiber MOS subsystem provides the MEGARA dispersed mode and allows placing 100 seven-fiber minibundles (92 in the MOS-dedicated pseudo-slit and 8 in the LCB pseudo-slit for sky background measurements) anywhere in the $3.5 \mathrm{x}$ $3.5 \operatorname{arcmin}^{2} \mathrm{FOV}$.

The positioning of each robot is done by two rotations. The interpolation between the two rotations allows the full area assigned to each positioner to be patrolled. The combination of both movements covers a circle with a radius of $11.605 \mathrm{~mm}$ from the centre of the positioner (this circle reaches the corners of the hexagon with an E/C of $20.1 \mathrm{~mm}$ ). The system is able to place the fibers in polar coordinates as showed in Fig. 13.

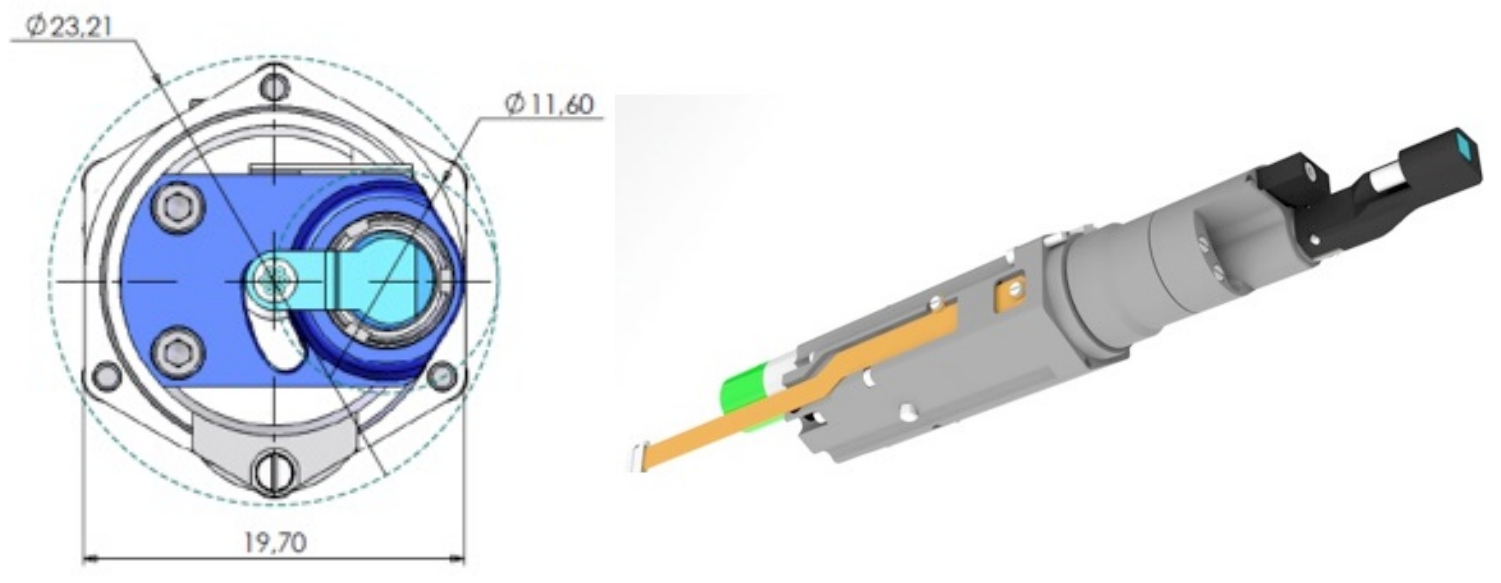

Fig. 13. Patrol area covered by each positioner (left). Full view of the positioner (right)

The main characteristics of the fiber positioner are listed below:

- Distance between positioners: $20.1 \mathrm{~mm}$

- Hexagonal distribution (it has been assessed as the best distribution for the application)

- Covered area: a circular area of $23.21 \mathrm{~mm}$ in diameter

- 2 Rotation interpolation, closed loop

- Rotation 1 range: $365^{\circ}$

- Rotation 2 range: $180^{\circ}$

- Positioning accuracy: $\pm 25 \mu \mathrm{m}$

- $\mathrm{Z}$ max defocus error: $\pm 200 \mu \mathrm{m}$

- Max angular tilt: $\pm 3 \operatorname{mrad}\left( \pm 0.19^{\circ}\right)$

- Maximum torque: $10 \mathrm{mNm}$

- Reconfiguration time: $10 \mathrm{sec}$ (for each individual positioner), $60 \mathrm{sec}$ (for the Fiber MOS)

- Weight $<95 \mathrm{~g}$

- Voltage: $3.3 \mathrm{~V}$

- Consumption $<200 \mathrm{~mA} / \mathrm{h}$ 
This fixing system shall be strong and repetitive. The fixing of each positioner has been designed considering that it must be possible to disassemble it from its back in order to facilitate the maintenance of the mechanical and electronics parts. The length of the power and signal cables and the fibers shall be also defined taking into account the need to assemble and disassemble each positioner independently.

The button, which is the mechanical part where the fibers are attached, has been defined taking into account the assembly of the microlenses and fibers, optical considerations and possible micro machining issues. The microlens arrays will be square shaped and include two reference marks to properly align the array with the fibers. However, a cylindrical shape for the button is suggested in order to adjust and assemble the microlens to the positioner arm. This geometry allows an exact X-Y positioning of the lenses and have also defined the position and tolerances of the microbores on the button and cross-shaped reference marks.

Fig. 14 shows a sketch of this component.

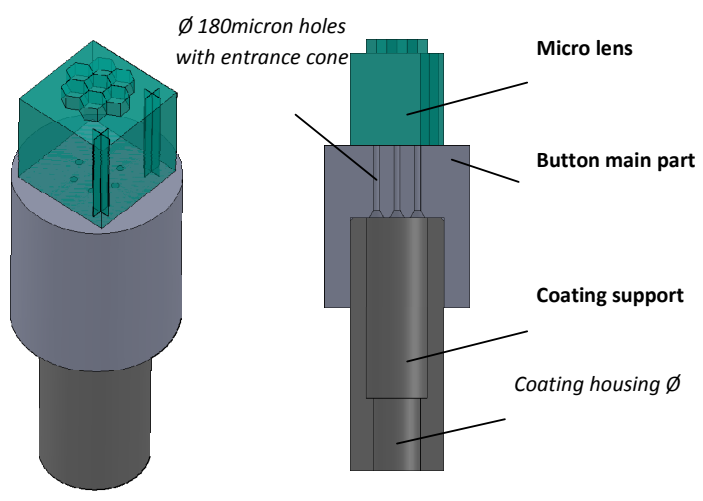

Fig. 14. Positioner button detailed design.

Regarding the positioner electronics, it has been decided to use a stepper motor with incremental encoders (as the initial choice of DC motors and absolute encoders is not now available for the required size) for R1 and a stepper motor with a Hall sensor for R2. In addition, hard stops shall be provided in both rotations to determine a zero position for the positioner.

Each positioner will have its own electronic card (PCB). Robot positioner electronics operates in a master-slave mode. Each robot positioner shall be connected to the MEGARA Control System LCU (that shall act as master controller) through a CANBUS network to ensure reliable communications and compliance with the GTC standard procedures.
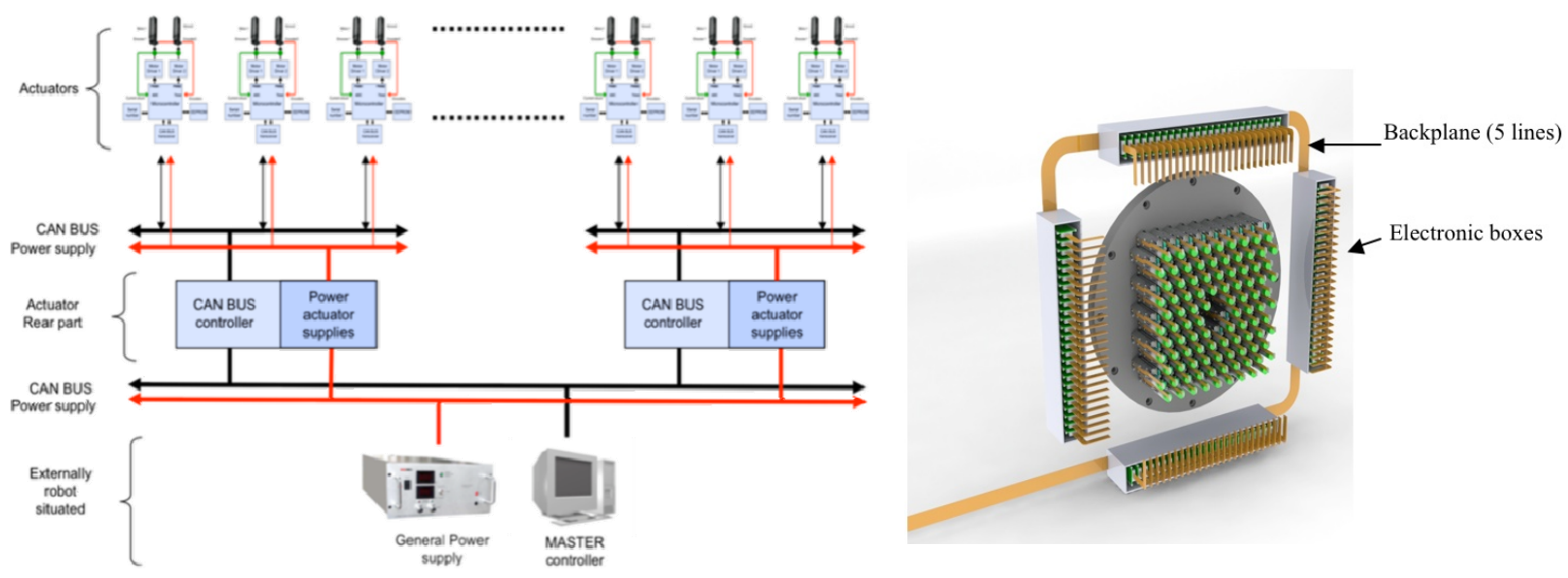

Fig. 15. Overall view of the positioner electronics architecture. 


\subsection{Pseudo-slit}

The fibers will be arranged in a pseudo-slit configuration (the pseudo-slit plates) and placed in front of each spectrograph at the pseudo-slit position. The pseudo-slit plates for all configuration modes shall be identical to allow a standard interface at the pseudo-slit position.

The focal plane at the pseudo-slit position is smoothly curved with a ROC of $1075 \mathrm{~mm}$ and a size (tangential to the curve) of $119 \mathrm{~mm}$. All fibers shall be mounted at the pseudo-slit, buffered side by side, forming a regularly spaced linear array. The fiber outer diameter $(170 \mu \mathrm{m}=$ allows achieving approximately the requested gap between consecutive fibers. This assembly in-line is acceptable since the scientific requirements of having 2 projected pixels on detector between adjacent fibers to reduce cross-talking effects is equivalent to have a pitch of $178 \mu \mathrm{m}$, so that having $170 \mu \mathrm{m}$ is fully acceptable.

As the polishing of the fiber mounted in a curved surface is not advisable, the decision was to split the pseudo-slit frame in several flat frames (where fibers are attached), called boxes, arranged to follow the curvature of the pseudo-slit, as shown in Fig. 16.

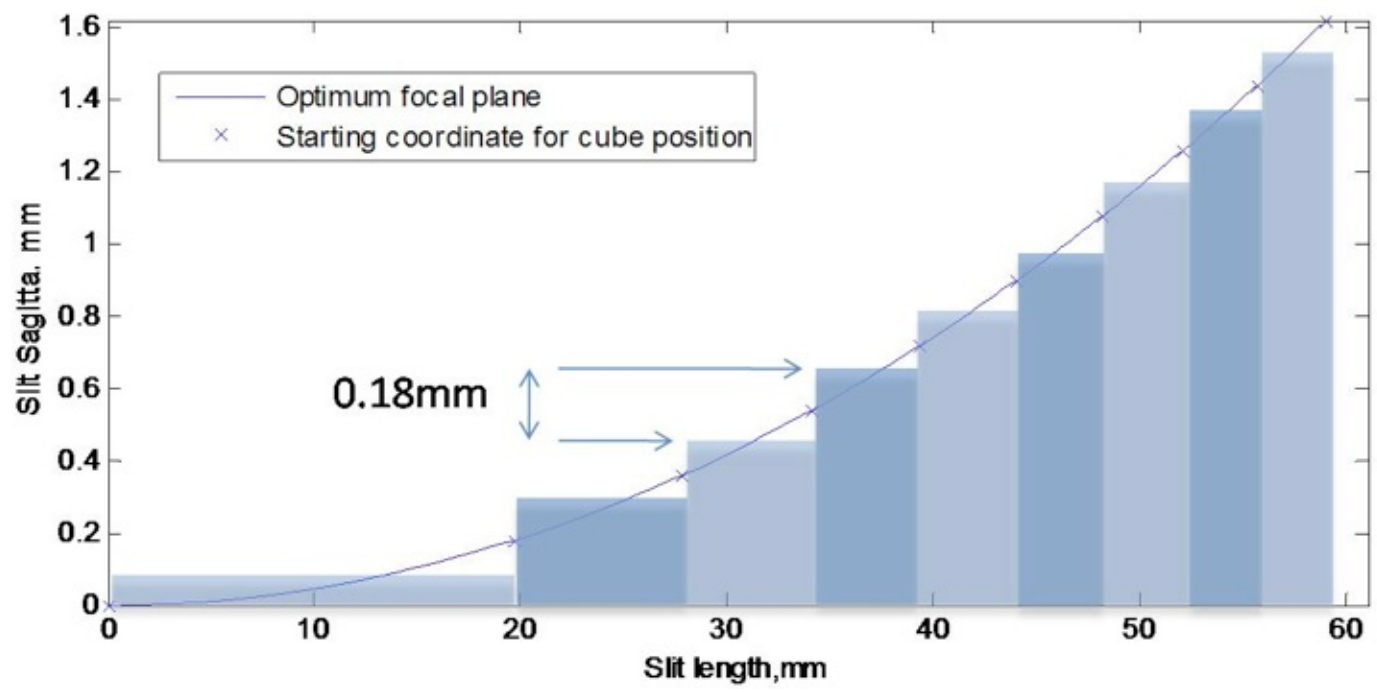

Fig. 16. Pseudo-slit boxes.

The number of fibers in each box (and therefore the longitudinal dimension D) shall be different in each box in order to reproduce the pseudo-slit curvature. There is a limited performance degradation (less than $4 \mathrm{~mm}$ from the curved pseudo slit case) that is inside the Image quality requirement.

In LCB case, the 623 fibers tube will have an octopus distribution and will be divided in dedicated tube benches that will arrive to each box in the LCB pseudo-slit. A similar solution is proposed for the SCB. In the MOS case, the fiber minibundles shall be organized in multiples of 7 fibers to be able to fit an exact number of positioners per box.

\section{MEGARA PRELIMINARY DESIGN PROTOTYPES}

This section provides an overview of the two prototypes that have been manufactured during the preliminary design: the Fiber bundle prototype and the Fiber MOS positioner prototype.

\section{- Fiber Bundle prototype}

The Fiber Bundle prototype includes a minibundle of 7 fibers $40 \mathrm{~m}$ in length (to simulate the current estimation of the length between the Folded Cassegrain focus and the spectrograph location at the GTC). This prototype ends on one side (the Folded Cassegrain Focal Station end) by a standalone positioner button (without the 
positioner) over which the microlens array shall be mounted and, on the other side (the spectrograph position), by a replica of a pseudo slit box (same design as the whole spectrograph pseudo slit although the size has been reduced in order to accommodate 7 fibers only).

Fibers have been already integrated at both ends by SEDI and it is only pending for integration of the microlens array (manufactured by AMUS) at the button end.

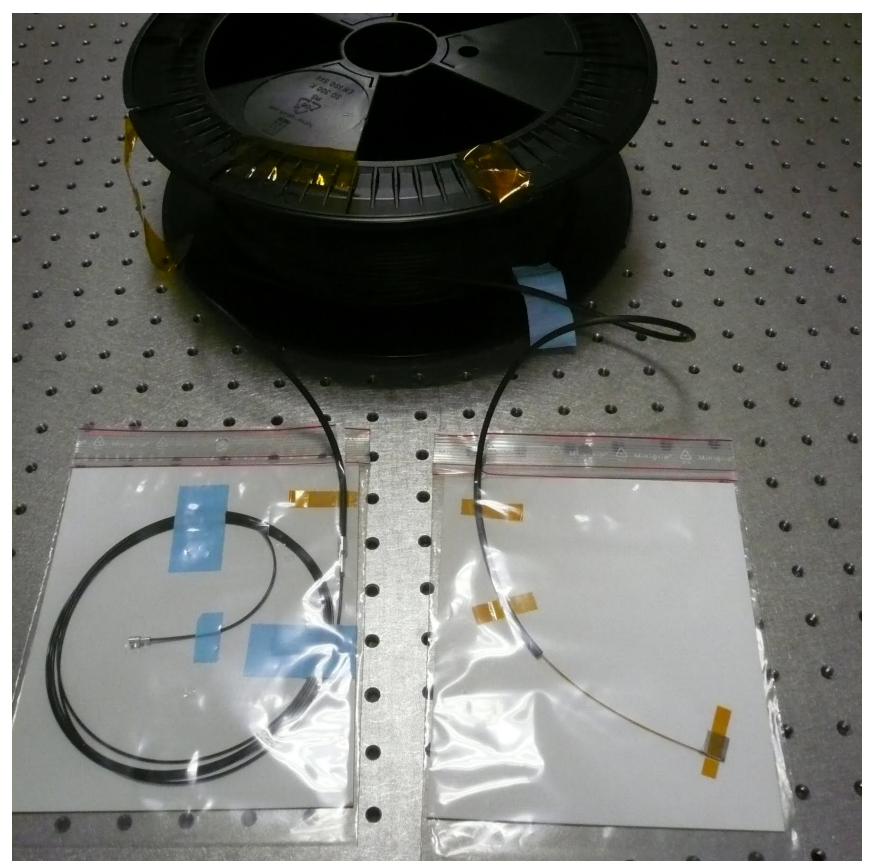

Fig. 17. Fiber Bundle prototype

This prototype will be used mainly to carry out several optical tests, as FDR measurements, in order to check the real optical behaviour of the entier fiber system (fibers and microlenses). These tests will be performed at LICA laboratory at the Complutense University (LICA-UCM).

It is also proposed to integrate this prototype at GTC in order to repeat these measurements on the GTC when $\mathrm{F} / \mathrm{C}$ rotator will be installed.

\section{- Fiber MOS positioner prototype}

The Fiber MOS positioner prototype includes a complete Fiber MOS Positioner and the 7-fiber minibundle attached to the positioner. The length of the fiber link is also $40 \mathrm{~m}$.

The positioner was designed and manufactured at AVS and then was sent to SEDI, where the fiber minibundle was integrated.

The Fiber MOS prototype is being used to perform mechanical, thermal and performance tests in order to check that the Fiber MOS requirements are achieved. These tests have been performed at the AVS company facilities (see Fig. 18).

Afterwards, this prototype will be shipped to LICA-UCM, where a microlens array will be integrated in order to use also this prototype to carry out optical test, including the dynamical measurement of the FRD of the system. This second battery of tests will be performed at LICA-UCM. 


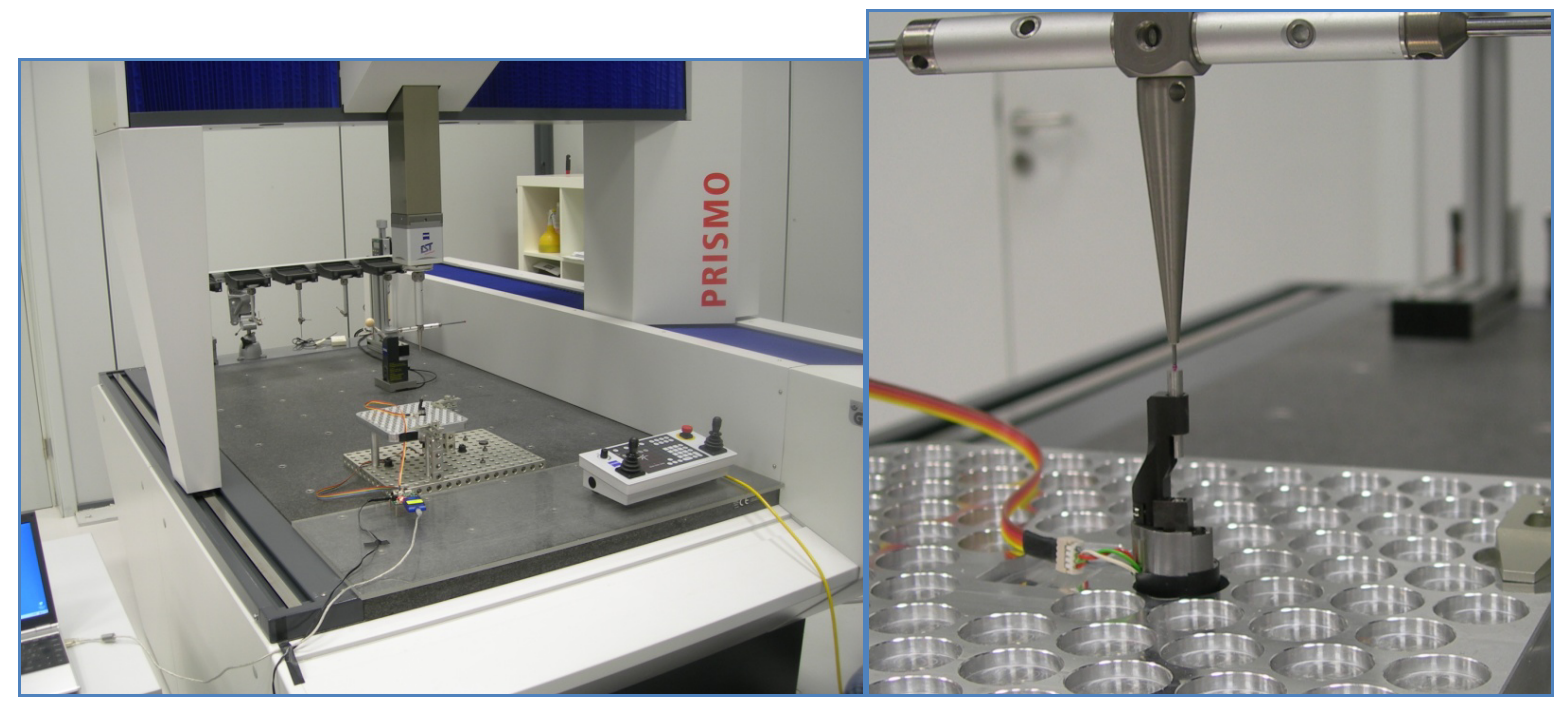

Fig. 18. Fiber MOS positioner prototype measurement bench.

Geometrical tests have been carried out to determine the behavior of both rotations (R1 and R2). All requirements (radius, flatness, parallelism, eccentricity, tilt and positioning accuracy) have been fulfilled. In particular, the worst-case positioning errors (at the outer diameters) were $11.4 \mu \mathrm{m}$ for R1 and $7.6 \mu \mathrm{m}$ for R2.

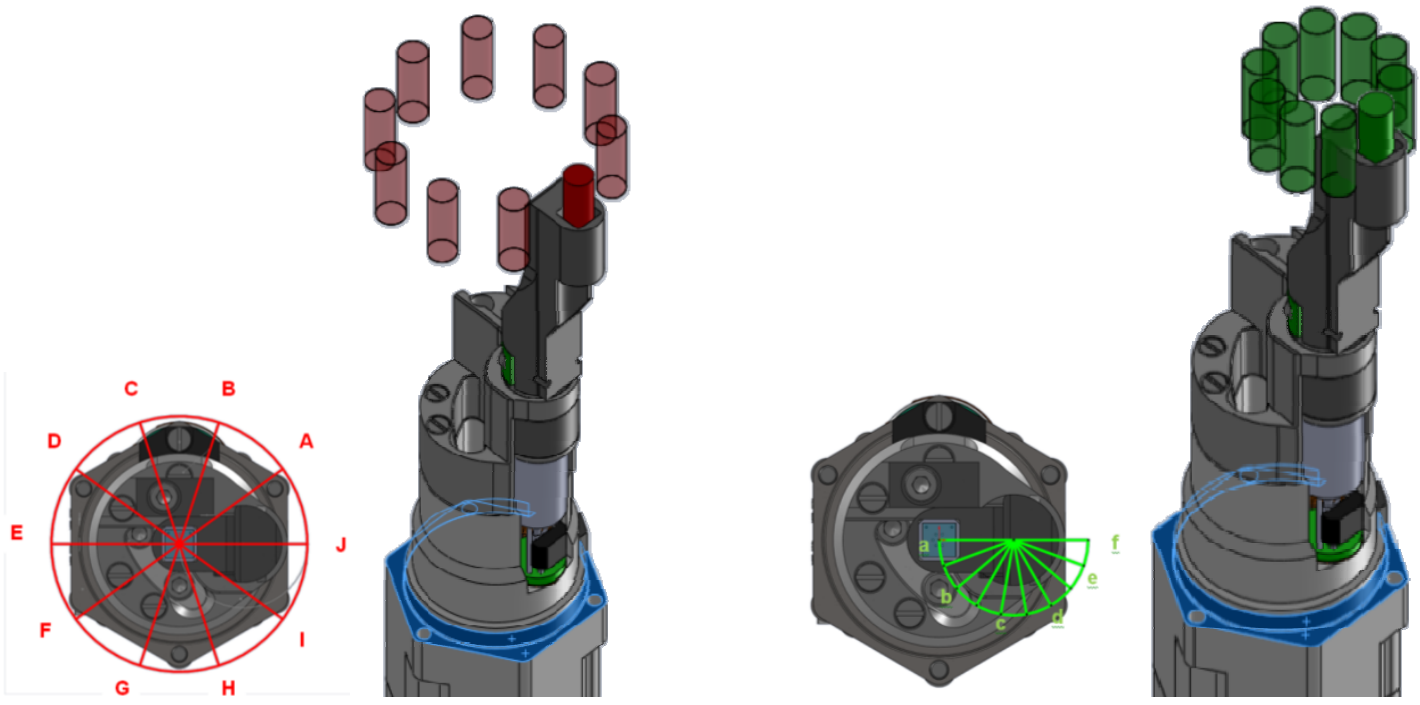

Fig. 19. R1 and R2 rotation measurement at their outer diameters.

Additional tests have been performed to check the Zero protocol, to measure the step uniformity, backlash and target repeatability. It was found that the backlash effect is negligible in both motions and that the maximum positioning difference with respect to the first measurements were $16.2 \mu \mathrm{m}$ for $\mathrm{R} 1$ and $2.7 \mu \mathrm{m}$ for $\mathrm{R} 2$. Therefore, we can conclude that the positioner prototype achieves the requirements providing high repeatability and high positioning accuracy.

All measurements have been performed at $21^{\circ}$ temperatures and in one unit only. The measurements on an additional unit and covering a wider temperature range were recommended to gain information about the system and will be implemented in the future. 


\section{CONCLUSIONS}

The current design of the MEGARA Focal Plane subsystems is presented in this paper as well as a summary of the status of the Fiber Bundle and Fiber MOS positioner prototypes that have manufactured during the preliminary design phase.

The design of the MEGARA Focal Plane subsystems presented in this paper has already evolved from the design presented at PDR as the MEGARA team has included a number of recommendations agreed with GRANTECAN and the PDR External Review Panel. Now the system is ready to enter into the detailed design.

Regarding the Fiber MOS positioner prototype, a full battery of test has been performed that allows us to conclude that the positioner fulfils the initial requirements. Additional tests will be carried out in an additional unit and in a wider range of temperatures.

Measurements on the Fiber Bundle prototype are still pending. The test rigs to allow the fibers to be accurately attached to the microlens arrays are currently being defined as well as the test bench to characterize the fiber link.

\section{REFERENCES}

[1] Gil de Paz, A, et Al., "MEGARA: the future optical IFU and multi-object spectrograph for the $10.4 \mathrm{~m}$ GTC telescope", Proc. SPIE 8446-182, (2012). 